\section{Functional Imaging to Differentiate Pulmonary Carcinoids}

TO THE EDITOR: We read with great interest the article by Kayani et al. (1). They evaluated 11 cases of typical carcinoids, 2 of atypical carcinoids, 1 of small cell neuroendocrine tumor, 1 of large cell neuroendocrine tumor, 2 of diffuse idiopathic pulmonary neuroendocrine hyperplasia, and 1 of adenocarcinoma with neuroendocrine differentiation using ${ }^{18} \mathrm{~F}-\mathrm{FDG}$ PET/CT and ${ }^{68} \mathrm{Ga}-$ DOTATATE PET/CT. They reported that, compared with typical carcinoids, atypical and other less differentiated carcinoids revealed significantly higher uptake on ${ }^{18} \mathrm{~F}$-FDG PET/CT scans $(P=0.005)$ and significantly lower uptake on ${ }^{68} \mathrm{Ga}$-DOTATATE PET/CT scans $(P=0.002)$.

We observed an interesting finding by evaluating the ratios of maximum standardized uptake value (SUVmax) on ${ }^{68} \mathrm{Ga}$-DOTATATE PET/CT to SUVmax on ${ }^{18} \mathrm{~F}-\mathrm{FDG}$ PET/CT scans. The ratios range from 1.2 to 19.5 in typical carcinoids (median SUVmax, 7.3) but from 0.1 to 1.1 in other less differentiated carcinoids (median SUVmax, 0.16). This difference in the ratios of typical versus atypical and other less differentiated carcinoids is statistically significant. The $P$ value is 0.002 (Mann-Whitney test for nonparametric data, 2-tailed). Thus, the ratio of SUVmax on ${ }^{68} \mathrm{Ga}-$ DOTATOC PET/CT and ${ }^{18}$ F-FDG PET/CT may also be of help in predicting the histopathologic variety of the carcinoid tumor and may have an equally high accuracy. A larger study is indicated to validate this observation and to objectively determine a cutoff value for the possible differentiation.

\section{REFERENCE}

1. Kayani I, Conry BG, Groves AM, et al. A comparison of ${ }^{68} \mathrm{Ga}$-DOTATATE and ${ }^{18}$ F-FDG PET/CT in pulmonary neuroendocrine tumors. J Nucl Med. 2009;50: 1927-1932.

\author{
Tarun Jindal \\ Arvind Kumar* \\ Rakesh Kumar \\ Roman Dutta \\ *Department of Surgery \\ All India Institute of Medical Sciences \\ New Delhi 110029, India \\ E-mail: arvindreena@gmail.com
}

DOI: 10.2967/jnumed.110.077826

REPLY: We thank the authors for their interest in our article (1). The authors state that the standardized uptake value ratios of ${ }^{68} \mathrm{Ga}$-DOTATATE PET/CT to ${ }^{18} \mathrm{~F}$-FDG PET/CT can differentiate well-differentiated from less differentiated carcinoids and predict the histopathologic variety of the carcinoids. However, caution should be exercised. Occasionally, well-differentiated and poorly differentiated cell populations exist within the same tumor mass,

COPYRIGHT @ 2010 by the Society of Nuclear Medicine, Inc. and predicting histology on the basis of ${ }^{68} \mathrm{Ga}$-DOTATATE PET/ CT-to- ${ }^{18}$ F-FDG PET/CT ratios would not be appropriate.

\section{REFERENCE}

1. Kayani I, Conry BG, Groves AM, et al. A comparison of ${ }^{68} \mathrm{Ga}$-DOTATATE and ${ }^{18}$ F-FDG PET/CT in pulmonary neuroendocrine tumors. J Nucl Med. 2009;50: $1927-1932$.

\section{Irfan Kayani}

Jamshed B. Bomanji*

*Institute of Nuclear Medicine

University College Hospital

235 Euston Rd.

London, U.K., NW1 2BU

E-mail: jamshed.bomanji@uclh.nhs.uk

DOI: 10.2967/jnumed.110.078527

\section{Cellular Dosimetry Using the Geant4 Monte Carlo Toolkit}

TO THE EDITOR: Cai et al. (1) reported Monte Carlo calculations to estimate cellular doses using ${ }^{111} \mathrm{In}$ in different cell configurations. Therefore, the authors used the Monte Carlo $\mathrm{N}$-particle (MCNP) code and compared their results with values provided by Goddu et al. $(2,3)$ for a single-cell model. Their results were within $66.2 \%-153.4 \%$ of the results of Goddu et al.

We performed similar simulations for a single-cell model using the Geant4 tool kit (4). Using the low-energy extensions, Geant4 is able to simulate electron-photon interactions down to $250 \mathrm{eV}$. The Geant4 source code is freely available and can be downloaded from the official Geant4 collaboration Web site (www.geant4. org). According to cellular experiments in our laboratory, we considered ${ }^{99 \mathrm{~m}} \mathrm{Tc},{ }^{123} \mathrm{I}$, and ${ }^{111} \mathrm{In}$. Emission spectra were taken from Howell (5); unlike Cai et al., we used electron and photon emissions. Decay sites were assumed to be homogeneously distributed inside the nucleus or cytoplasm or on the cell surface; dose deposition was considered only in the nucleus for different cell and nucleus radii. For each run, $10^{7}$ particles were simulated to give at least 10,000 hits in the nucleus.

Our results corresponded well to the $S$ values given by the authors in their Table 1. For a single cell, $\mathrm{S}$ values $\mathrm{S}(\mathrm{N} \leftarrow \mathrm{N})$ are $0.3 \%-3 \%$ higher, $\mathrm{S}(\mathrm{N} \leftarrow \mathrm{Cy})$ deviates from $-1.3 \%$ to $10.1 \%$, and $\mathrm{S}(\mathrm{N} \leftarrow \mathrm{CS})$ deviates from $-5.1 \%$ to $7.4 \%(\mathrm{~N}, \mathrm{Cy}$, and $\mathrm{CS}$ are nucleus, cytoplasm, and cell surface, respectively).

Furthermore, Cai et al. calculated $\mathrm{S}$ values for cell clusters and cell monolayers. These values are important when cells in those configurations were irradiated. Thus far, we have examined clonogenic cell survival, whereby cells can be considered as single cells. Hence, we do not calculate $S$ values for clusters or monolayers. Nevertheless, the compliance between the results of Cai et al. (1), Goddu et al. (2), and our simulations show the feasibility of using Monte Carlo methods to assess absorbed doses in cellular dimensions. In contrast to MCNP, Geant4 is free and is available on the Web. 